DOI: 10.12957/demetra.2017.22435

\title{
Nível de satisfação e escolhas alimentares dos comensais em restaurante self-service
}

\section{Satisfaction level and food choices of diners in a self-service restaurant}

\author{
Michele Honicky' \\ Kelín Schwarz \\ Renata Leia Demario Vieira? \\ Priscila Lumi Ishii Freire \\ Raquel Rosalva Gatti? \\ 1 Universidade Estadual do Centro-Oeste, \\ Departamento de Nutrição, Guarapuava-PR, Brasil. \\ Correspondência / Correspondence \\ Michele Honicky \\ E-mail: michele_honicky@yahoo.com.br
}

\section{Resumo}

Nos últimos anos, houve um aumento da alimentação fora de casa, juntamente com a alta concorrência nos serviços de alimentação e a preocupação com a saúde dos comensais. O objetivo do presente estudo foi avaliar a satisfação dos comensais quanto aos serviços oferecidos e suas escolhas alimentares em um restaurante self-service. A amostra do estudo totalizou 148 indivíduos, os quais avaliaram os serviços prestados em relação aos seguintes critérios: aparência dos alimentos, apresentação do bufê, variedade, temperatura das saladas, sobremesas e pratos quentes, sabor das refeições, tempero, atendimento, higiene e limpeza do local e avaliação geral. A satisfação dos comensais em relação ao serviço prestado, no geral, teve avaliação positiva, indicando bom grau de contentamento. Em relação às escolhas alimentares, verificou-se grande influência dos fatores aparência, sabor, variedade, forma de preparo, valor nutricional e alimentos que fazem bem à saúde. A avaliação da satisfação e as escolhas alimentares dos comensais para restaurantes self-service são ferramentas que ajudam a determinar o perfil dos comensais e a realidade da unidade, visando melhorar os serviços prestados.

Palavras-chave: Serviços de Alimentação. Comportamento do Consumidor. Comportamento Alimentar. Hábitos Alimentares.

\section{Abstract}

In recent years, there has been an increase in the habit of eating out, as well as stronger competition in the food service and more concern for diners" health. The aim of the study was to evaluate 
diners' satisfaction with the services offered and their food choices in a self-service restaurant. The study sample had 148 individuals. They evaluated the services based on the following criteria: food appearance; buffet presentation; variety, temperature of salads, desserts and hot meals; taste of meals; seasoning; service; hygiene and cleanliness of the premises and overall assessment. Diners' satisfaction with the overall service provided had a positive evaluation, indicating a good satisfaction level. Their food choices were influenced by factors such as visual appearance, taste, variety, method of preparation, nutritional value and healthy foods. The evaluation of diners' satisfaction and food choices in self-service restaurants are tools that help determine the profile of diners and the conditions of the self-service restaurant, with a view to improving the services provided.

Key words: Food Services. Consumer Behavior. Feeding Behavior. Food Habits.

\section{Introdução}

Em decorrência das mudanças nos padrões de vida e comportamentos alimentares, nos últimos anos ocorreu um aumento da alimentação fora de casa, em virtude de diversos fatores, como o aumento da participação feminina no mercado de trabalho, falta de tempo para o preparo de refeições, melhora do nível de educação, flexibilidade nos horários para se alimentar e variedade de alimentos. ${ }^{1}$

No Brasil, verificou-se crescimento no gasto com alimentação fora de casa de 22,2\% entre 2002/2003 para 27,9\% entre 2008/2009, o que significa diferença de 5,9\% em seis anos e com probabilidade de aumento nos próximos anos. ${ }^{2}$

Os restaurantes self-service são comuns no Brasil, neles o comensal escolhe o que deseja consumir, pagando o equivalente ao peso dos alimentos que colocou em seu prato. ${ }^{3}$ Dessa maneira, ele tem a liberdade de escolha dos alimentos de acordo com suas preferências e variedade de oferta, influenciado por vários motivos, como aparência, valor nutricional, sabor, preço, variedade, higiene, além de questões biológicas, socioculturais, antropológicas e econômicas. ${ }^{4}$

A busca por variedade e facilidade leva os comensais a fazer refeições fora de casa, porém os mesmos relatam preocupação com a sua qualidade nutricional. ${ }^{5}$ Entretanto, a alimentação fora de casa pode atender tanto à necessidade da praticidade quanto à de uma refeição saudável, ainda mais quando associada à educação nutricional, oferta de preparações salutares e informações nutricionais para os comensais, levando-os a escolhas alimentares adequadas. ${ }^{6}$ 
Em razão da alta concorrência nesse tipo de estabelecimento- a avaliação da satisfação dos comensais é importante para verificar a necessidade de mudanças e resolução dos problemas encontrados. ${ }^{7}$ A satisfação é aferida pela qualidade nutricional, variedade ofertada, ambiente, tempo de espera, textura dos alimentos e sabor das refeições. Portanto, o contentamento do comensal depende de esses critérios atingirem ou superarem suas expectativas. ${ }^{8}$

Diante do aumento da alimentação fora de casa e a preocupação com a alimentação equilibrada, é importante satisfazer às expectativas dos comensais, tanto na questão da qualidade dos serviços prestados, o que inclui uma alimentação saudável, quanto da oferta de alimentos de acordo com o perfil do consumidor. Nesse sentido, o objetivo do estudo foi avaliar a satisfação dos comensais quanto aos serviços oferecidos e suas escolhas alimentares em um restaurante self-service.

\section{Metodologia}

Trata-se de um estudo descritivo, para compreender e descrever o comportamento de adultos e adolescentes de um restaurante comercial self-service, com características quantitativas. O método utilizado foi um estudo de caso, que, segundo Pereira, ${ }^{9}$ caracteriza-se pela análise detalhada de um objeto específico, com o propósito de aprofundar a compreensão de determinado caso. A realização de um estudo de caso nesse restaurante self-service é importante para analisar a qualidade dos serviços oferecidos e conhecer as escolhas alimentares dos comensais de forma a determinar detalhadamente o perfil dos comensais e a realidade do restaurante, a fim de aperfeiçoar os serviços prestados por meio de estratégias para satisfazer às expectativas e preferências dos comensais. $\mathrm{O}$ estudo foi realizado com adultos e adolescentes que frequentam um restaurante comercial selfservice da cidade de Guarapuava-PR, de ambos os sexos, que aceitaram voluntariamente participar do estudo e assinaram o Termo de Consentimento Livre e Esclarecido.

Os dados foram coletados durante quatro dias, sendo abordados por conveniência, aproximadamente, 50 participantes por dia. Após se servirem no bufê, os comensais foram convidados a participar do estudo voluntariamente. Em caso de aceite, os participantes recebiam as orientações sobre o questionário e o preenchiam após o almoço, entregando-o na saída do local.

Para avaliação do nível de satisfação, foi utilizado questionário adaptado de Proença, ${ }^{10}$ que classifica nove critérios de satisfação aos serviços oferecidos, classificando-os em excelente/ótimo, bom, satisfatório e ruim, por meio de um escala de 1 a 10, sendo considerado excelente, de 10 a 9; bom, de 8 a 7; satisfatório, de 6 a 5 ; e ruim, abaixo de 5 pontos. Os critérios foram relacionados à aparência dos alimentos, apresentação do bufê, variedade, temperatura das saladas, sobremesas e pratos quentes, sabor das refeições, tempero, atendimento, higiene e limpeza do local e avaliação geral. A soma do porcentual da classificação excelente/ótimo e bom dos critérios, quando acima de $70 \%$, indica um bom nível de satisfação dos comensais aos serviços prestados. 
A avaliação das escolhas alimentares ocorreu por meio de questionário de escolhas alimentares adaptado de Jomori, ${ }^{4}$ o qual forneceu informações sobre as práticas alimentares, os fatores e motivos de determinadas escolhas alimentares e o que os comensais participantes julgam ser uma alimentação saudável. O questionário foi adaptado para adolescentes com a finalidade de facilitar o preenchimento.

Os dados foram tabulados no Excel ${ }^{\circledR}$, analisados de forma descritiva- em médias, desvio-padrão, frequências e porcentagens. A comparação entre os resultados referentes ao que os consumidores colocam no prato e o que acham que deveriam colocar foi realizada por meio do Teste do Quiquadrado. Todas as análises foram realizadas com auxílio do Software SAS versão 9.0, com nível de significância de $5 \%(\mathrm{p}<0,05)$.

A pesquisa foi aprovada no Comitê de Ética em Pesquisa (COMEP) da Universidade Estadual do Centro-Oeste (UNICENTRO), sob o parecer de número 840.222/2014.

\section{Resultados}

Participaram da pesquisa 200 comensais, porém 47 recusaram e cinco questionários foram excluídos devido ao preenchimento incompleto. Sendo assim, a amostra total consistiu de 148 participantes, com média de idade de $27,35( \pm 14,10)$ anos, e destes, $58,78 \%$ do sexo feminino. A maioria dos indivíduos declarou não ter problema de saúde. E os que declararam ter apresentaram hipertensão arterial, rinite e esteatose hepática.

A maioria dos participantes prefere realizar as refeições acompanhada e não se preocupa com a sua imagem corporal na hora da escolha dos alimentos. Em relação à frequência com que almoça no restaurante, a maior parte faz esta refeição de duas a três vezes por semana, de quatro a cinco vezes por semana e eventualmente (Tabela 1). 
Tabela 1. Idade (média \pm desvio-padrão), sexo, frequência de refeições no restaurante, problemas de saúde e preferências no momento de se alimentar de indivíduos que frequentam um restaurante comercial self-service. Guarapuava, PR. 2015.

\begin{tabular}{llc}
\hline Idade & & $27,35 \pm 14,10$ \\
Sexo & Masculino & $41,22(61)$ \\
Frequência que almoça & Feminino & $58,78(87)$ \\
no restaurante & $4-5$ vezes por semana & $24,32(36)$ \\
$\%$ (n) & $2-3$ vezes por semana & $32,43(48)$ \\
& 1 vez por semana & $9,46(14)$ \\
& Eventualmente & $24,32(36)$ \\
Problema de saúde & Raramente & $9,46(14)$ \\
(n) & Não apresenta & $95,95(142)$ \\
& Hipertensão & $2,70(4)$ \\
Prefere se alimentar & Rinite & $0,68(1)$ \\
(n) & Esteatose hepática & $0,68(1)$ \\
Aparência influencia & Sozinho & $8,78(13)$ \\
nas escolhas & Acompanhado & $91,22(135)$ \\
\hline
\end{tabular}

Notas: $\%=$ porcentagem; $\mathrm{n}=$ número amostral.

Na tabela 2 estão os resultados encontrados na pesquisa de avaliação do nível de satisfação. Dos critérios avaliados, os que indicaram bom nível de satisfação foram limpeza do ambiente, atendimento, aparência dos alimentos, apresentação do bufê, temperatura das saladas/sobremesas, tempero e sabor das refeições. Observa-se que o estabelecimento apresenta bom nível de satisfação dos comensais.

No entanto, notou-se número relevante de comensais que avaliaram como "satisfatório" variedade, temperatura dos pratos quentes, sabor das refeições e tempero. A temperatura dos pratos quentes também apresentou classificação "ruim" para alguns comensais.

No geral, a maioria dos comensais classificou o serviço prestado como bom. 
Tabela 2. Nível de satisfação $(\%, \mathrm{n})$ dos indivíduos que frequentam um restaurante comercial self-service. Guarapuava, PR. 2015.

\begin{tabular}{|c|c|c|c|c|}
\hline \multirow[b]{2}{*}{ Critérios } & \multicolumn{4}{|c|}{ Classificação do nível de satisfação } \\
\hline & $\begin{array}{c}\text { Excelente/Ótimo } \\
\%(\mathrm{n})\end{array}$ & $\begin{array}{l}\text { Bom } \\
\%(n)\end{array}$ & $\begin{array}{c}\text { Satisfatório } \\
\%(\mathrm{n})\end{array}$ & $\begin{array}{l}\text { Ruim } \\
\%(\mathrm{n})\end{array}$ \\
\hline Aparência dos alimentos & $52,03(77)$ & $36,49(54)$ & $11,49(17)$ & $0(0)$ \\
\hline Apresentação do bufê & $39,19(58)$ & $47,97(71)$ & $12,84(19)$ & $0(0)$ \\
\hline Variedade & $31,76(47)$ & $38,51(57)$ & $27,03(40)$ & $2,70(4)$ \\
\hline $\begin{array}{l}\text { Temperatura das } \\
\text { saladas / sobremesas }\end{array}$ & $32,43(48)$ & $54,05(80)$ & $10,14(15)$ & $3,38(5)$ \\
\hline $\begin{array}{l}\text { Temperatura dos } \\
\text { pratos quentes }\end{array}$ & $29,05(43)$ & $38,51(57)$ & 23,65 (35) & $8,78(13)$ \\
\hline Sabor das refeições & $38,51(57)$ & $41,89(62)$ & $19,59(29)$ & $0(0)$ \\
\hline Tempero & $35,81(53)$ & $45,27(67)$ & $16,89(25)$ & $2,03(3)$ \\
\hline Atendimento & $63,51(94)$ & $27,70(41)$ & $8,11(12)$ & $0,68(1)$ \\
\hline $\begin{array}{l}\text { Higiene e limpeza do } \\
\text { ambiente }\end{array}$ & $60,81(90)$ & $35,14(52)$ & $4,05(6)$ & $0(0)$ \\
\hline $\begin{array}{l}\text { No geral, como você } \\
\text { avalia nosso serviço }\end{array}$ & $38,51(57)$ & $54,73(81)$ & $6,76(10)$ & $0(0)$ \\
\hline
\end{tabular}

Notas: \%= porcentagem; $\mathrm{n}=$ número amostral.

As escolhas alimentares dos comensais estão descritas na tabela 3. Os fatores que mais influenciam essa decisão são a questão da aparência, variedade, sabor e odor e forma de preparo, e os motivos mais citados que levam à escolha desses alimentos são: "fazem bem à saúde", "porque raramente como eles em casa" e "porque estão com aparência boa" .

A maioria dos comensais relatou que frequenta o restaurante porque confia na higiene do local. Também relataram que não pegam algo de aparência ruim no bufê e não se servem de maionese ou carne suína, devido ao medo de contaminação e/ou intoxicação alimentar. As principais preferências de pratos dos comensais são: preparações que agradam o paladar, preparações diferentes que não consomem em casa e comida caseira (Tabela 3). 
Tabela 3. Fatores $(\%, n)$ que influenciam nas escolhas alimentares dos indivíduos que frequentam um restaurante comercial self-service da cidade de Guarapuava, PR. 2015.

$$
\text { Fatores que influenciam na escolha alimentar } \% \text { (n) }
$$

\section{Para escolher a comida que colocará no prato}

Aparência

$74,32(110)$

Sabor e odor

Forma de preparo

Variedade

Valor nutricional

Preço

Algo novo para provar

Algo que não engorde

\section{Motivos que levou a escolher esses alimentos}

Faz bem à saúde/ sustenta

$85,14(126)$

Não quero engordar / quero ganhar peso

$37,16(55)$

Foi conselho profissional / para tratamento de problema de saúde

Pesam menos no prato, portanto pago menos

Porque raramente os como em casa

Porque estavam com uma "cara boa"

\section{Algo que não colocaria no prato}

Só escolho o que costumo comer em casa, porque eu sei como é feito

$10,81(16)$

Não pego salada ou qualquer coisa cru, não sei se está bem lavada

$6,76(10)$

Não pego maionese ou carne de porco, tenho medo de intoxicação / contaminação

Como de tudo, não me importo com a higiene, o importante é o sabor

Se eu vou num lugar, confio

Não pego algo de aparência ruim

Não pego algo que me faça engordar 


\section{Pratos que têm preferência}

Comida caseira

Preparações diferentes que não costumo comer em casa

Preparações raras que só como em festas/comemorações

Preparações que agradam meu paladar

Notas: $\%=$ porcentagem; $\mathrm{n}=$ número amostral.

Na tabela 4, observa-se a comparação entre o que os comensais consideram uma refeição de costume e uma refeição saudável no restaurante self-service. Ressalta-se que no que consideram refeição saudável há diminuição com diferença significante de alimentos fritos, sobremesa, massas, batata, carne bovina $(\mathrm{p}<0,001)$ e arroz $(\mathrm{p}=0,02)$ em relação ao prato de costume. Por outro lado, na refeição tida como saudável houve aumento significante dos que citaram salada $(\mathrm{p}=0,011)$. 
Tabela 4. Comparação dos alimentos que os indivíduos que frequentam um restaurante comercial self-service costumam colocar no prato versus alimentos que deveriam colocar no prato $(\%, \mathrm{n})$ para uma alimentação saudável. Guarapuava, PR. 2015.

\begin{tabular}{lccc}
\hline & $\begin{array}{c}\text { Alimentos que } \\
\text { costumam colocar } \\
\text { no prato } \\
\%(\mathrm{n})\end{array}$ & $\begin{array}{c}\text { Alimentos que } \\
\text { deveriam colocar } \\
\text { no prato \% (n) }\end{array}$ & $\mathrm{p}^{\mathrm{a}}$ \\
\hline Salada & $\begin{array}{c}\text { 81,08 (120) } \\
\text { Arroz }\end{array}$ & $91,22(135)$ & 0,011 \\
Feijão & $85,14(126)$ & $74,32(110)$ & 0,020 \\
Massas & $69,59(103)$ & $74,32(110)$ & 0,365 \\
Batata & $54,73(81)$ & $15,54(23)$ & $<0,001$ \\
Carne bovina & $41,89(62)$ & $18,24(27)$ & $<0,001$ \\
Carne branca (frango/peixe) & $50,68(75)$ & $24,32(36)$ & $<0,001$ \\
Alimentos fritos & $72,50(107)$ & $70,27(104)$ & 0,699 \\
Alimentos assados e & $30,41(45)$ & $6,08(9)$ & $<0,001$ \\
grelhados & $63,51(94)$ & $67,57(100)$ & 0,463 \\
Sobremesa & $61,49(91)$ & $13,51(20)$ & $<0,001$ \\
\hline
\end{tabular}

Notas: ${ }^{a} \mathrm{p}=$ valor estatístico do teste (Qui quadrado) \%= porcentagem; $\mathrm{n}=$ número amostral.

\section{Discussão}

A avaliação do nível de satisfação e das escolhas alimentares dos comensais tem o objetivo de apresentar informações para o desenvolvimento de ações que visem melhorar a qualidade do serviço prestado na unidade. Uma vez identificado o grau de satisfação dos comensais, pode-se elaborar planos que proporcionem melhores condições de atendimento aos comensais, principalmente nos critérios com baixa avaliação. ${ }^{11}$

No geral, os resultados encontrados no presente estudo indicam bom nível de satisfação dos comensais. Resultados semelhantes foram encontrados no estudo de Feil, ${ }^{12}$ que verificou avaliação positiva do nível de satisfação dos comensais em uma Unidade de Alimentação e Nutrição aos serviços prestados. Assim como no estudo de Araújo et al., ${ }^{13}$ cuja avaliação dos comensais variou de 89,6\% a 95,3\% para os parâmetros ótimo e bom nos aspectos ambiente, higiene do restaurante, atendimento, variedade, sabor e apresentação das refeições. 
No entanto, em alguns atributos, como a temperatura dos pratos quentes, a unidade do presente estudo recebeu maior percentual de avaliações negativas, o que merece atenção, uma vez que as preparações no bufê em temperatura inadequada podem contribuir para multiplicação de micro-organismos e riscos de infecções e intoxicações alimentares. ${ }^{14}$ Nesse sentido, destaca-se a importância de seguir a resolução RDC número 216, que indica que os alimentos devem ser mantidos em temperatura superior a $60^{\circ} \mathrm{C}$ por no máximo 6 horas para garantir a segurança e qualidade higiênico-sanitária. ${ }^{15}$ Em estudo de Ramos et al, ${ }^{16}$ observou-se que os comensais que almoçavam no final da distribuição também avaliavam negativamente a temperatura dos alimentos, o que pode indicar aquecimento inadequado do bufê. Em contrapartida, no estudo de Bessa ${ }^{17}$ nenhum comensal estava insatisfeito com a temperatura dos alimentos.

Entre os critérios avaliados no estudo, além da temperatura dos pratos quentes, o sabor das preparações, o tempero e variedade também apresentaram alto percentual de respostas de classificação "satisfatória" e "ruim" em comparação com os outros critérios. Percebe-se a importância de buscar estratégias para satisfazer esses comensais, pois a variedade dos alimentos ofertada está relacionada com a qualidade do serviço e o sabor das preparações auxilia na obtenção e manutenção dos comensais, além de diminuir os índices de desperdício e aumentar o lucro da unidade. ${ }^{18}$ Entretanto, em estudo realizado em Unidade de Alimentação e Nutrição em Pelotas verificou-se 46,2\% de avaliações insatisfatórias para a variedade de alimentos e 38,6\% para o sabor das preparações, percentuais estes maiores que os observados na presente pesquisa. ${ }^{19}$

A avaliação da satisfação não envolve apenas aspectos do alimento em si, mas atendimento, ambiente e valor da refeição. ${ }^{20}$ Dessa maneira, a avaliação do serviço no geral recebeu maior percentual de respostas na classificação "bom”, com destaque para o atendimento, aparência dos alimentos e limpeza do ambiente, com a maioria das respostas "excelente". A avaliação positiva da unidade pode ser justificada por um adequado planejamento, organização, direcionamento e supervisão da unidade. ${ }^{21}$

Nos últimos anos, observa-se o aumento da concorrência dos restaurantes self-service. ${ }^{7}$ Por isso, as unidades produtoras de refeições devem procurar diferentes maneiras de satisfazer e manter seus clientes, visto isto, uma opção pode ser verificar as escolhas alimentares dos comensais para caracterizar o seu perfil e complementar as informações sobre a avaliação do nível de satisfação para melhoria dos serviços prestados.

Quanto aos fatores para a escolha dos alimentos, verificou-se que parte dos comensais considera importante a aparência, sabor, variedade dos alimentos, forma de preparo e valor nutricional para colocar o alimento no prato. Em estudo de Jomori et al, ${ }^{22}$ constatou-se que os principais critérios que influenciaram a escolha dos alimentos foram a aparência do alimento, forma de preparo, saúde associada à imagem corporal e o hedonismo, resultados semelhantes ao presente estudo. 
Visto que no presente estudo o restaurante recebeu avaliação positiva para atendimento e limpeza do ambiente, entende-se que tais quesitos também sejam fatores associados com a seleção dos alimentos. Em geral, os principais motivos que influenciam a escolha do restaurante são variedade de comidas e bebidas, atendimento, ambiente interno do restaurante, aspectos sensoriais, alimentação saudável e nutritiva, formas de pagamento, ${ }^{23}$ limpeza do local, higiene dos funcionários e qualidade dos alimentos. ${ }^{19}$

O tipo de serviço também influencia a preferência pelos alimentos. Em serviço do tipo self-service, geralmente, observam-se escolhas mais saudáveis quando comparadas com as no serviço à la carte, ${ }^{24}$ e também há menor possibilidade de excessos, pois os comensais pagam pelo peso do alimento. ${ }^{6}$

De acordo com os comensais do presente estudo, o principal motivo que os leva a escolher os alimentos que colocam no prato é "porque fazem bem para saúde". Em estudo com resultados semelhantes, verificou-se que os comensais relataram ser a saúde o mais frequente motivo que influencia a escolha dos alimentos. ${ }^{25}$ Outra pesquisa que avaliou as influências nas práticas alimentares dos comensais também observou a preocupação com a saúde. ${ }^{26}$ Sendo assim, possivelmente a escolha dos alimentos pode estar ligada com a preocupação com a saúde.

Entretanto, outro estudo verificou que a alimentação saudável não predomina na escolha alimentar, esta é ocasional, pois outros aspectos estão envolvidos na escolha dos alimentos, como valor, gosto e hábitos alimentares. ${ }^{6}$ Nesse sentido, a opção pelos alimentos é influenciada por outros motivos, como na presente pesquisa, no caso de "porque estavam com uma aparência boa", "porque não quero engordar", "porque raramente os consumo em casa" e "porque pesam menos no prato, portanto pago menos".

Ainda em relação às escolhas alimentares dos comensais deste estudo, verifica-se a confiança dos mesmos no aspecto higiene. No entanto, apenas um pequeno percentual relata medo de contaminação ou intoxicação alimentar, e por isso não se serve do prato de maionese ou carne de porco. Outro estudo também indicou que os fatores de riscos de contaminação alimentar não apresentaram significância nas escolhas alimentares. ${ }^{4}$

Por outro lado, alto percentual de comensais responderam não se servir de algo com aparência ruim, evidenciando que os aspectos sensoriais estão entre os principais fatores que determinam a escolha alimentar, ${ }^{27}$ semelhante ao presente estudo, que verificou ser a aparência o principal fator para colocar ou não algo no prato.

Quanto à comparação da refeição de costume e o que colocariam no prato para uma refeição saudável, observa-se que a maioria põe no prato alimentos fritos, massas e sobremesas, que são preparações com maior densidade energética. O excesso do consumo de alimentos fritos, massas e doces está associado com o desenvolvimento da obesidade. ${ }^{28}$ Além disso, no Brasil constatou-se que a alimentação fora de casa apresenta relação positiva com o sobrepeso e obesidade em homens. ${ }^{29}$ 
No presente estudo também se observou que os comensais têm conhecimento de que um prato saudável deve conter salada, porém na maioria dos casos esta não se encontra presente no prato de costume. O incentivo ao aumento do consumo de produtos in natura, como verduras, legumes e frutas, para promoção da saúde é largamente conhecido e sustentado inclusive pelo Guia Alimentar para a População Brasileira. ${ }^{30}$ Não obstante, um estudo que comparou tipos de restaurante constatou que os restaurantes do tipo self-service aumentam o consumo de frutas e verduras e diminuem o consumo de alimentos com alta densidade energética. ${ }^{26}$ Dessa forma, considera-se que o comensal do restaurante self-service tem a possibilidade de se servir de acordo com suas preferências alimentares e a variedade de alimentos ofertados, permitindo-se escolhas alimentares saudáveis ou não saudáveis.

\section{Conclusão}

O restaurante self-service avaliado tem bom nível de satisfação dos comensais, principalmente em relação ao atendimento, aparência dos alimentos e limpeza do ambiente. Por outro lado, a temperatura dos pratos quentes foi avaliada por alguns comensais como satisfatória e ruim. Além disso, com a aplicação do questionário de escolhas alimentares, observa-se que os comensais recebem influência da aparência, variedade, sabor e odor dos alimentos na escolha dos alimentos, bem como "daqueles alimentos que fazem bem a saúde".

Dessa maneira, por meio da avaliação do nível de satisfação e escolhas alimentares, é possível identificar os critérios avaliados negativamente e implementar estratégias específicas para correção, a fim de satisfazer às expectativas dos comensais e conhecer o seu perfil de escolha alimentar, de forma a oferecer alimentos seguindo essas preferências.

Por fim, ressalta-se que ambas as avaliações são ferramentas úteis para ajudar a determinar o perfil dos comensais e a realidade da unidade, visando aperfeiçoar os serviços prestados.

\section{Colaboradores}

Honicky M e Schwarz K participaram da concepção e desenho do estudo, da análise e interpretação dos dados e elaboração do artigo. Honicky M, da coleta de dados. Vieira RLD, Freire PLI e RR Gatti RR da revisão crítica de conteúdo.

Conflito de Interesses: Os autores declaram não haver conflito de interesses. 


\section{Referências}

1. Leal D. Crescimento da alimentação fora do domicilio. Segurança Alimentar e Nutricional 2010; 17(1):123-132.

2. Claro RM, Baraldi LG, Martins APB, Bandoni DH, Levy RB. Evolução das despesas com alimentação fora do domicílio e influência da renda no Brasil, 2002/2003 a 2008/2009. Cad. Saúde Pública 2014; 30(7):1418-1426.

3. Abreu ES, Torres EAFS. Restaurante "por quilo": vale o quanto pesa? Uma avaliação do padrão alimentar em restaurantes em São Paulo. Nutrire: Rev Soc Bras Aliment Nutr. 2003; 25(1):7-22

4. Jomori MM. Escolhas alimentares do comensal em um restaurante por peso. [Dissertação]. [Florianópolis]: Universidade Federal de Santa Catariana, Pós Graduação de Nutrição; 2006.

5. Avelar SEA. Fatores que influenciam no consumo de alimentos fora do lar. [Dissertação]. [Lavras]: Universidade Federal de Lavras, Pós Graduação Administração; 2010.

6. Santos MV, Proença RPC, Fiates GMR, Calvo MCM. Os Restaurantes por peso no contexto de alimentação saudável fora de casa. Rev Nutr. 2011; 24(4):641-649

7. Nobre PA. Avaliação da satisfação dos clientes em restaurante do tipo self-service em Brasília. [Especialização]. [Brasília]: Centro de Excelência em Turismo de Brasília; 2004.

8. Meister SPA. Pesquisa de satisfação dos clientes do restaurante vermelho grill [Trabalho de conclusão de curso]. [Porto Alegre]: Departamento de Ciências Administrativas, Universidade Federal do Rio Grande do Sul; 2008.

9. Pereira M. Epidemiologia: teoria e prática. Rio de Janeiro: Guanabara Koogan; 1995.

10. Proença RPC, Veiros MB. Sistemas de qualidade nutricional, sensorial e simbólica na produção de refeições. In: Vieira MNCM, Japur CC. Gestão de qualidade na produção de refeições. Rio de Janeiro: GEN; 2012. p. 205-216.

11. Proença RPC, Sousa AA, Veiros MB, Hering B. Qualidade nutricional e sensorial na produção de refeições. Florianópolis: EdUFSC; 2005. 221 p.

12. Feile CC, Adami FS, Bosco SMD, Fassina P. Pesquisa de satisfação dos comensais de uma unidade de alimentação e nutrição. Revista UNINGÁ 2015; 43(1):32-35.

13. Araújo AC, Chiapetta DMS, Correia RC. Satisfação de clientes em relação ao serviço de nutrição de um restaurante. Nutrire: Rev Soc Bras Aliment Nutr. 2011; 36(Suplemento 11o Congresso Nacional da Sociedade Brasileira de Alimentação e Nutrição):98.

14. Alves MG, Ueno M. Restaurantes self-service: segurança e qualidade sanitária dos alimentos servidos. Rev Nutr. 2010; 23(4):573-580.

15. Brasil. Agência Nacional de Vigilância Sanitária. Resolução. RDC n. 216, 15 de setembro de 2004. Dispõe sobre regulamento técnico de boas práticas para serviços de alimentação. Diário Oficial da União 16 set. 2004.

16. Ramos SA, Souza de FR de, Fernandes GCB; Xavier SKP. Avaliação qualitativa de cardápio e pesquisa de satisfação em uma unidade de alimentação e nutrição. Braz J Food Nutr. 2013; 24(1):29-35. 
17. Bessa AP, Araújo MBV. Análise da satisfação de clientes do serviço de nutrição de uma Unidade de Alimentação e Nutrição de Uberaba MG. Cadernos de Pós-Graduação da Fazu 2011; 2(1):1-5.

18. Vaz CS. Restaurantes: controlando custos e aumentando lucros. Viçosa: UFV; 2006.

19. Gardin ETO, Cruvinel EBS. Avaliação da satisfação dos clientes do restaurante universitário (RU) do campus Londrina da Universidade Tecnológica Federal do Paraná. [Trabalho de conclusão de curso]. [Londrina]: Universidade Tecnológica Federal do Paraná; 2014.

20. Meller RFO, Nunes NS, Santos LP, Schäfer AA, Leal CMA. Avaliação da satisfação de clientes em relação ao serviço de nutrição de uma unidade de alimentação e nutrição da cidade de Pelotas (RS). Anais do Congresso de Iniciação Científica; Encontro de Pós-Graduação, Primeira Mostra Científica; 2009; Pelotas. Pelotas: UFPEL; 2009. p.1-4

21. Abreu ES, Spinelli MGN, Araújo RMV. Fatores de risco ambiental para trabalhadores de unidade de alimentação e nutrição. Nutrição em Pauta 2002; 57(53):46-49.

22. Jomori MM, Proença RPC, Calvo MCM. Determinantes de escolha alimentar Rev Nutr. 2008; 21(1):63-73.

23. Souki GQ, Madureira KT, Gonçalves FC, Reis NMT. Desenvolvimento e validação de uma escala de atributos utilizados pelos consumidores na escolha de restaurantes de comida a quilo. Revista de Administração da Unimep 2010; 8(2):72-90.

24. Castelo BNSD. Análise da alimentação fora do domicílio de consumidores do centro comercial do Município do Rio de Janeiro - RJ [Tese]. [Campinas]: Faculdade de Engenharia de Alimentos da Universidade Estadual de Campinas; 2000.

25. Connors M, Bisogni CA, Sobal J, Devine CM. Managing values in personal food systems. Appetite 2001;36(3):189-200.

26. Lassen A, Hansen K, Trolle E. Comparison of buffet and à la carte serving at worksite canteens on nutrient intake and fruit and vegetable consumption. Public Health Nutr. 2007;10(3):292-7.

27. Von Atzingen MCBC, Pinto E, Silva MEM. Sensory characteristics of food as a determinant of food choices. Nutr.: J Brazilian Soc Food Nutr. 2010; 35(3):183-196.

28. Sichieri R. Dietary patterns and their associations with obesity in the Brazilian city of Rio de Janeiro. Obes Res. 2002; 10(1):42-8.

29. Bezerra IN, Sichieri R. Eating out of home and obesity: a Brazilian nationwide survey. Public Health Nutr. 2009; 12(11):2037-43.

30. Brasil. Ministério da Saúde. Secretaria de Atenção à Saúde. Departamento de Atenção Básica. Guia alimentar para a população brasileira. $2^{a}$ ed. Brasília: MS; 2014.

Recebido: 12/4/2016

Revisado: 25/10/2016

Aceito: 21/2/2017 